\title{
POR QUE OTIMIZAÇÃO MULTIVARIADA?
}

\section{WHY MULTIVARIATE OPTIMIZATION?}

\section{Patricio Peralta-Zamora}

Doutor em Ciências pela UNICAMP. Professor do Departamento de Química da Universidade Federal do Paraná.

Coordenador do grupo de pesquisa na área de novas tecnologias para tratamento de resíduos

\section{JosMaRIa LOPES DE MORAIS}

Mestre em Ciências pelo CEFET-PR. Professora do Centro Federal de Educação Tecnológica do Paraná e doutoranda do Departamento de Química da UFPR na área de tratamento de resíduos

\begin{abstract}
NOEMI NAGATA
Doutora em Ciências pela UNICAMP. Professora do Departamento de Química da Universidade Estadual de Ponta Grossa, onde desenvolve pesquisa na área de Calibração Multivariada
\end{abstract}

Recebido: 19/07/04 Aceito: 22/05/05

\section{RESUMO}

Normalmente, estudos que objetivam a otimização de variáveis experimentais são realizados através de procedimentos que avaliam o efeito de uma variável por vez, o que, de maneira geral, impede o estabelecimento de ótimos verdadeiros, em razão da freqüência com que as variáveis se apresentam altamente correlacionadas. Neste trabalho, algumas aplicações práticas são apresentadas, visando demonstrar a conveniência dos procedimentos multivariados de otimização, principalmente sistemas de planejamento fatorial de experimentos. A partir de três exemplos práticos relacionados com a otimização de processos de degradação, discutem-se aspectos relacionados com o desenho experimental e a interpretação de resultados.

PALAVRAS-CHAVE: Otimização experimental, análise multivariada, planejamento fatorial.

\begin{abstract}
Studies aiming at the optimization of experimental variables are generally carried out by procedures that evaluate the effect of just one variable on time. However, this approach does not allow the establishment of true optimal values, mainly due to close connections that may exist among the studied variables. In this paper some practical applications based upon factorial design systems are used to demonstrate the convenience of multivariate procedures. The three examples described here entail the optimization of degradation processes related to the experimental design as well as interpretation of results.
\end{abstract}

KEYWORDS: Experimental optimization, multivariate analysis, factorial design.

\section{INTRODUÇÃO}

Provavelmente, a otimização de parâmetros experimentais de relevância seja uma das etapas mais críticas do trabalho científico, principalmente daqueles que objetivam o desenvolvimento de processos tecnológicos aplicáveis em grande escala.

Quais as variáveis relevantes? Quais os valores que devem ser ensaiados? Qual a ordem de estudo das variáveis? Qual a melhor resposta a ser analisada? Como desenvolver um trabalho de otimização, com o mínimo de trabalho experimental?

Diante desta problemática, cada pesquisador tem a sua forma de enfrentar este trabalho, normalmente apoiado na experiência acumulada na área. Entretanto, é importante esclarecer que na maioria dos casos, o processo de otimização é realizado de maneira univariada. Isto é, utilizando-se o clássico sistema de uma variável por vez. Obviamente, este tipo de trabalho, que envolve um grande número de experimentos, pode fornecer condiçōes que permitem um valor otimizado da resposta. No entanto, por negligenciar a interação entre as variáveis, o resultado obtido não necessariamente corresponde às condiçốes que levam ao ótimo verdadeiro. A explicação é simples. Nos sistemas químicos, as variáveis costumam se correlacionar fortemente, interagindo através de mecanismos que proporcionam efeitos sinérgicos e antagônicos. Se este fato é ignorado, o processo de otimização apresenta pouco valor.

Nos últimos anos, os sistemas multivariados de otimização têm ganhado bastante força, demonstrando a sua utili- dade nos mais variados campos do conhecimento. Dentro deste contexto, destaquem-se os estudos envolvendo processos biotecnológicos (Dueñas et al, 2003), sínteses orgânicas (Kochana et al, 2003), psicologia (Sharp e Hargrove, 2004), processos químicos (Montes, et al, 2004; Hardy et al, 2004) e, o que nos parece mais importante, otimização de processos industriais (Galetakis e Raka, 2004; Yi-zeng et al, 2001).

Dentre as várias alternativas existentes, destaque pode ser dado aos sistemas de planejamento fatorial, os quais permitem avaliar simultaneamente o efeito de um grande número de variáveis, a partir de um número reduzido de ensaios experimentais. A teoria em que esses sistemas se baseiam é bastante simples e está disponível na literatura especializada (Bruns et al, 2001). Para entendimento 
dos fundamentos, recomenda-se a leitura do material didático disponibilizado no site do chemkeys (http:// www.chemkeys.com $/ \mathrm{bra} / \mathrm{md} /$ peeo_ 6/mdoeq_1/mdpf_3/mdpf_3.htm).

O presente trabalho pretende demonstrar a conveniência dos sistemas de planejamento fatorial, recorrendo a exemplos práticos e reais relacionados com estudos de otimização em sistemas químicos de complexidade variável.

\section{METODOLOGIA}

Os exemplos apresentados correspondem estudos de remediação de substratos aquosos de relevância ambiental (licor negro de Kraft, chorume de aterro sanitário e corantes reativos), utilizando-se processo $\mathrm{UV} / \mathrm{H}_{2} \mathrm{O}_{2}$, coagulação com cloreto férrico e sistema redutivo com ferro metálico, respectivamente.

Todos os ensaios foram realizados em escala de bancada, utilizando-se reagentes de grau analítico e reatores convencionais.

Para controle analítico utilizaram-se determinações de fenóis totais, demanda química de oxigênio e descoloração, todas aplicadas de acordo com procedimentos padrão descritos na literatura (APHA, 1995).

\section{RESULTADOS E DISCUSSÃO}

\section{Primeiro caso}

O primeiro exemplo trata sobre um estudo de otimização de variáveis operacionais relevantes (concentração de peróxido de hidrogênio e $\mathrm{pH}$ ), visando a remediação de um resíduo de origem industrial (licor negro de Kraft) por processo oxidativo avançado (sistema $\mathrm{UV} / \mathrm{H}_{2} \mathrm{O}_{2}$ ). Como a influência de cada variável é estudada entre dois limites, o desenho corresponde a um planejamento fatorial $2^{2}$, descrito na Tabela 1 . Em um sistema deste tipo, apenas 4 ensaios são necessários para avaliar todas as combinaçôes possíveis. Entretanto, para uma melhor avaliação de tendências e da reprodutibilidade da metodologia analítica envolvida (determinação de fenóis totais pelo método de Folin-Ciocalteau), um ponto central ensaiado em triplicata foi incluído no estudo. É importante salientar que a seleção de variáveis a serem empregadas no estudo, os níveis em que estas serão ensaiadas e ainda a resposta utilizada para processar o planejamento devem ser criteriosamente estabelecidas, sendo que para tal, o conhecimento prévio do sistema em estudo é de fundamental importância.

De acordo com as informaçóes contidas na Tabela 1 , a única diferença entre os ensaios 1 e 2 está representada pela modificação da concentração de peróxido de hidrogênio. Desta forma, a diferença observada na resposta $(62-79=-17)$ indica que a degradação perde 17 pontos percentuais quando a concentração de peróxido muda do nível superior $(+)$ para o nível inferior (-), mantendo-se fixo o valor de $\mathrm{pH}$ no nível inferior (-). Uma análise similar permite calcular o efeito da concentração de peróxido quando o $\mathrm{pH}$ encontra-se fixo no nível superior (ensaios 3 e 4). Neste caso, o efeito (86-43 = 43) indica que ganhos de 43 pontos percentuais são introduzidas com a mudança da concentração de peróxido (do nível superior para o nível inferior). Para calcular o efeito médio, basta realizar a média aritmética entre ambos valores $[(-17+43) / 2=+13]$, o que define o efeito global apresentado pela variável em questão.

Considerando-se a variável $\mathrm{pH}$, cálculos similares podem ser feitos, encontrando-se os valores apresentados na Tabela 1. Um efeito global de -6 , indica que quando o $\mathrm{pH}$ é modificado do nível inferior para o superior a resposta monitorada sofre uma diminuição de 6 pontos percentuais.
Com base nestes antecedentes, e levando-se em consideração o desvio padrão típico da determinação envolvida ( 2 pontos percentuais), é possível concluir que ambas variáveis influenciam significativamente a resposta monitorada. Se a intenção fosse maximizar esta resposta, as condições escolhidas deveriam corresponder aos níveis inferiores de ambas variáveis (ensaio 1). Entretanto, pode-se observar que a melhor resposta não é dada pelas condiçōes do ensaio 1 , e sim pelas do ensaio 3 .

A explicação para este fato é bem simples e se origina na forte interação existente entre ambas as variáveis. Para uma melhor interpretação deste fenômeno, o planejamento é apresentado na forma de uma representação geométrica na Figura 1. Nesta representação, pode-se observar que o efeito do peróxido de hidrogênio não é o mesmo em ambos valores de $\mathrm{pH}$. Enquanto que o efeito é positivo (+17) para o menor valor de $\mathrm{pH}$ empregado, ele torna-se negativo (-43) no nível superior de $\mathrm{pH}$. Esta diferença, que também fica clara quando se avalia o efeito do $\mathrm{pH}$, somente pode ser explicada em termos de forte interação entre variáveis. $\mathrm{O}$ valor numérico associado a esta interação, denominado efeito de segunda ordem, pode ser calculado de acordo com o apresentado na Tabela 1.

Em situações de interação tão significativa quanto esta é que o sistema de planejamento fatorial demonstra o seu 


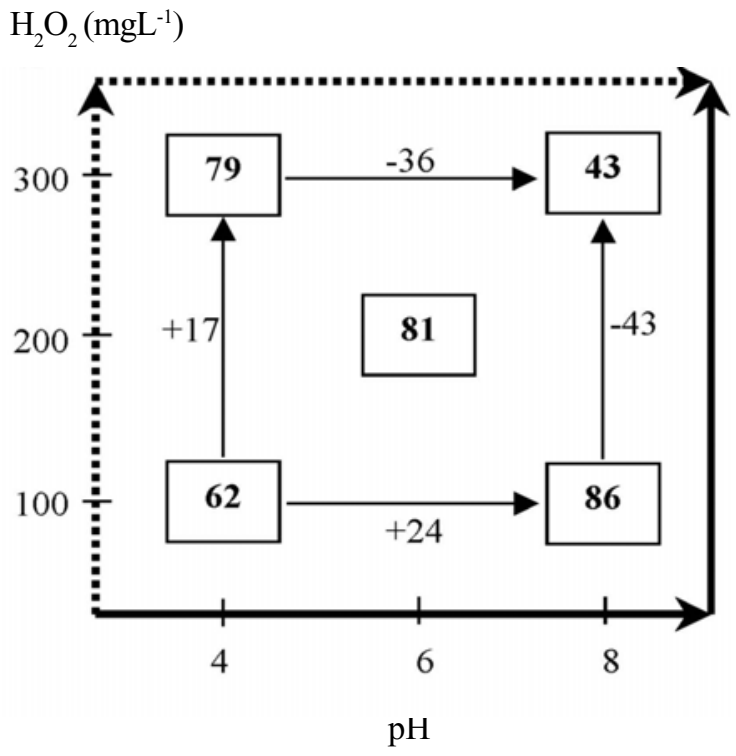

Figura I - Representação geométrica do planejamento fatorial 2 para otimização do sistema $\mathrm{H}_{2} \mathrm{O}_{2} / \mathrm{UV} \mathrm{na}$ remediação de licor negro de Kraft (Resposta: \% de degradação de fenóis totais em $\mathbf{3 0}$ min de tratamento)

valor. Se um procedimento de otimização univariado tivesse sido executado, seguindo a seqüência descrita palas setas pontilhadas, as condiçôes recomendadas seriam as do ensaio 2 , com uma eficiência de degradação otimizada de $79 \%$. Se o caminho descrito pelas setas sólidas tivesse sido escolhido, o valor otimizado real teria sido alcançado (ensaio 3), com eficiência de degradação de $86 \%$. Este é um dos exemplos que demonstra a capacidade do sistema de planejamento fatorial em avaliar as interaçôes possíveis, eliminando o caráter aleatório associado à escolha do caminho univariado.

Finalmente, é importante salientar que, embora o sistema permita evidenciar as interações entre as varáveis estudadas, a explicação deve ser fundamentada em argumentos técnicos relacionados com o processo em estudo. Neste caso, o favorecimento do processo de degradação em elevados valores de $\mathrm{pH}$ pode ser explicado em termos de favorecimento do processo de absorção de radiação, em função do maior coeficiente de absorção molar apresentado pelo ânion peroxila em $254 \mathrm{~nm}$ (Legrini et al, 1993). Por sua vez, o fato da eficiência ser fortemente reduzida para altas concentrações de peróxido, mesmo em $\mathrm{pH}$ alcalino, pode ser explicado pela recombinação de radicais hidroxila, típica de situaçôes de super produção de radicais (Equação 1).

$\mathrm{HO}^{\circ}+\mathrm{HO}^{\circ} \rightarrow \mathrm{H}_{2} \mathrm{O}_{2}$
$(+2,5)$, uma vez que se mostra inferior que os desvios típicos apresentados pela determinação de DQO (+/- 5\%). Por sua vez, o significativo efeito negativo do $\mathrm{pH}(-22,5)$ implica que maiores remoções de DQO podem ser conseguidas em baixos valores de $\mathrm{pH}$.

Entretanto, a existência de um importante efeito de segunda ordem (C x pH: -7,5) implica uma estreita correlação entre as variáveis. Diante deste tipo de correlação, o uso de aproximaçôes univariadas certamente poderia ter levado a conclusōes diferentes. Se o sistema univariado tivesse seguido o caminho descrito pelas setas pontilhadas (Figura 2), as condições otimizadas seriam as do ensaio 1, com 30\% de remoção de DQO. $\mathrm{O}$ ótimo verdadeiro somente teria sido alcançado, percorrendo-se o caminho descrito pelas setas sólidas.

Recentemente, um estudo de otimização similar foi reportado por Wang et al (2002), utilizando o sistema convencional que consiste no estudo de uma variável por vez. Na primeira etapa do estudo, o pH foi fixado em 7,6 (valor natural das amostras de chorume empregado) e a concentração de cloreto férrico foi modificada entre 100 e $1000 \mathrm{mg} \mathrm{L}^{-1}$. Utilizando-se a redução no teor de DQO solúvel como argumento, foi possível admitir que $500 \mathrm{mg} \mathrm{L}^{-1}$ representa a melhor dose de coagulante. Entretanto, cabe questionar se a melhor dose de cloreto férrico teria sido esta, se outro valor de $\mathrm{pH}$ tivesse sido selecionado. Em uma segunda etapa, os autores optaram por fi-

Tabela 2 - Planejamento fatorial $2^{2}$ para otimização das condições de remediação de chorume de aterro sanitário por processo de coagulação/floculação (Resposta: \% remoção de DQO)

\begin{tabular}{ccccc}
\hline Variável & Nível (-) & \multicolumn{2}{c}{ Nível (0) } & Nível (+) \\
[coagulante, C] $\mathrm{mg} \mathrm{L}^{-1}$ & 250 & \multicolumn{2}{c}{500} & 750 \\
$\mathrm{pH}$ & 5,1 & \multicolumn{2}{c}{7,0} & 9,1 \\
& \multicolumn{3}{c}{ Variáveis } \\
\cline { 2 - 4 } Experimento & $\mathrm{C}$ & $\mathrm{pH}$ & $\mathrm{C} \mathrm{x} \mathrm{pH}$ & Resposta \\
\cline { 2 - 5 } 1 & - & - & + & 30 \\
2 & + & - & - & 40 \\
3 & - & + & - & 15 \\
4 & + & + & + & 10 \\
5 & 0 & 0 & 0 & 17 \\
6 & 0 & 0 & 0 & 12 \\
7 & 0 & 0 & 0 & 22 \\
\hline
\end{tabular}

Efeitos principais: C: $(-30+40-15+10) / 2=+2,5+/-5$; $\mathrm{pH}:(-30-40+15+10) / 2=-22,5+/-5$ Efeito de segunda ordem: $\mathrm{C} \times \mathrm{pH}:(+30-40-15+10) / 2=-7,5+/-5$ 
xar a concentração de coagulante em $500 \mathrm{mg} \mathrm{L}^{-1}$, sendo o efeito do $\mathrm{pH}$ estudado entre os valores 3 e 8 . Uma vez que discretas diferenças na remoção de DQO foram observadas nesta etapa do estudo, a melhor escolha certamente estaria representada por valores de $\mathrm{pH}$ próximos do valor natural da matriz em estudo. Com base nestes resultados, as melhores condiçôes para a remoção de $\mathrm{DQO}$ em amostras de chorume não foram claramente determinadas.

A análise deste trabalho nos permitiu observar que após um grande número de experimentos univariados, não foi possível o estabelecimento de valor otimizado de resposta. Também podemos levantar a questão da ordem da escolha das variáveis, caso inicialmente tivesse sido fixado o valor da concentração de cloreto férrico e o $\mathrm{pH}$ fosse variado, quais seriam os resultados?

Obviamente, este tipo de trabalho, teria um encaminhamento diferente caso a interação entre as variáveis tivesse sido considerada. $\mathrm{O}$ estudo multivariado, especialmente para matrizes complexas, permite um encaminhamento muito melhor que o seu estudo univariado.

\section{Terceiro caso}

No terceiro exemplo apresenta-se um estudo que objetiva a otimização de três variáveis que influenciam um processo de degradação envolvendo ferro metálico. Trata-se, portanto, de um planejamento $2^{3}$, cuja configuração é apresentada na Tabela 3. Novamente um ponto central em triplicata foi adicionado, o que implica a necessidade de realizar 11 ensaios.

O valor positivo do efeito da concentração de peróxido de hidrogênio (+13) e ferro metálico (+ 40) implica que melhores condições de degradação são conseguidas com estas variáveis no seu nível superior. Contrariamente, o efeito negativo do $\mathrm{pH}(-35)$ implica que as melhores condiçôes estão representadas por valores de $\mathrm{pH}$ que tendem ao mínimo. Estas condiçóes correspondem às do ensaio 4, que permitem degradações maximizadas de $96 \%$.

A existência de significativos efeitos de segunda ordem implica uma elevada inter-relação entre as variáveis. Esta nada surpreendente relação, novamente demonstra que tentativas univariadas poderiam levar à obtenção de ótimos não verdadeiros, tal como pode ser observado pelo caminho descrito pelas setas ponti-

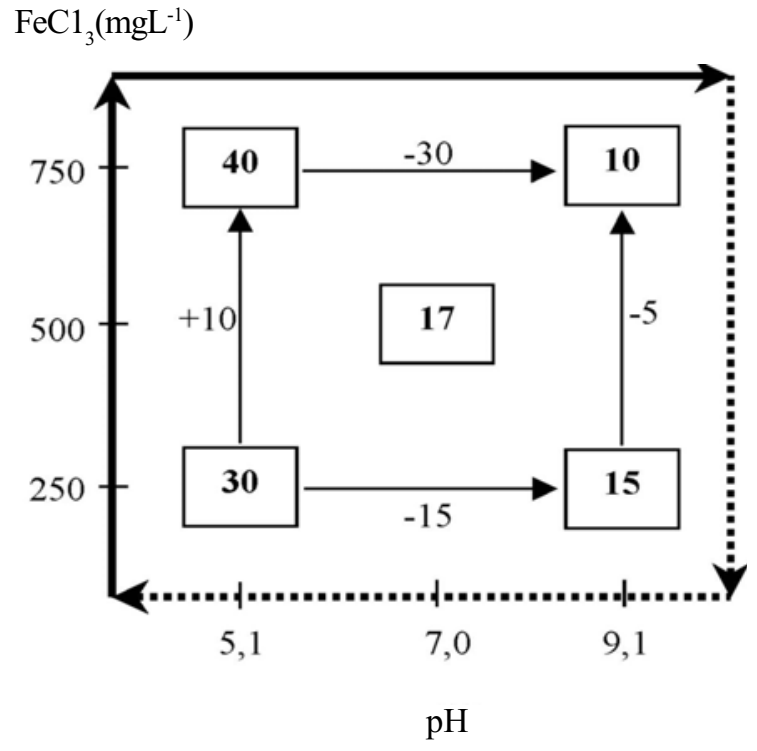

Figura 2 - Representação geométrica do planejamento fatorial $2^{2}$
para otimização das condições de remediação de chorume de
aterro sanitário por processo de coagulação/floculação
(Resposta: remoção de DQO)

Tabela 3-Planejamento fatorial $2^{3}$ para otimização das condições de degradação do corante Laranja Reativo I 6 por processo envolvendo ferro metálico

(Resposta: \% de degradação do corante avaliada por espectroscopia UV-Vis)

\begin{tabular}{|c|c|c|c|c|}
\hline & & \multicolumn{2}{|c|}{ Nível (0) } & \\
\hline $\mathrm{pH}$ & 2 & \multicolumn{2}{|c|}{3} & 4 \\
\hline$\left[\mathrm{H}_{2} \mathrm{O}_{2}\right] \mathrm{mg} \mathrm{L}^{-1}$ & 0 & \multicolumn{2}{|c|}{50} & 100 \\
\hline$\left[\mathrm{Fe}^{0}\right] \mathrm{g} \mathrm{L}^{-1}$ & 0 & \multicolumn{2}{|c|}{0,5} & 1,0 \\
\hline & \multicolumn{3}{|c|}{ Variáveis } & \multirow[b]{2}{*}{ Resposta } \\
\hline Experimento & $\mathrm{H}_{2} \mathrm{O}_{2}$ & $\mathrm{Fe}^{0}$ & $\mathrm{pH}$ & \\
\hline 1 & - & - & - & 3 \\
\hline 2 & + & - & - & 5 \\
\hline 3 & - & + & - & 56 \\
\hline 4 & + & + & - & 96 \\
\hline 5 & - & - & + & 1 \\
\hline 6 & + & - & + & 1 \\
\hline 7 & - & + & + & 5 \\
\hline 8 & + & + & + & 14 \\
\hline 9 & 0 & 0 & 0 & 86 \\
\hline 10 & 0 & 0 & 0 & 83 \\
\hline 11 & 0 & 0 & 0 & 85 \\
\hline
\end{tabular}

Efeitos principais: $\mathrm{H}_{2} \mathrm{O}_{2}:(-3+5-56+96-1+1-5+14) / 4=13+/-1,5$

$\left[\mathrm{Fe}^{0}\right]:(-3-5+56+96-1-1+5+14) / 4=40+/-1,5$

$\mathrm{pH}:(-3-5-56-96+1+1+5+14) / 4=-35+/-1,5$

Efeito de segunda ordem: $\mathrm{H}_{2} \mathrm{O}_{2} \times\left[\mathrm{Fe}^{0}\right]:(+3-5-56+96+1-1-5+14) / 4=12+/-1,5$

$\left[\mathrm{Fe}^{0}\right] \times \mathrm{pH}:(+3+5-56-96-1-1+5+14) / 4=-32+/-1,5$ 
lhadas, na representação geométrica apresentada na Figura 3.

\section{CONCLUSÕES}

Os resultados comentados neste trabalho demonstram claramente a inconveniência dos procedimentos usualmente utilizados em estudos de otimização de variáveis experimentais. Fundamentados na análise de variáveis isoladas, estes sistemas negligenciam os efeitos de interação, o que dificulta ou inviabiliza a reconhecimento de condiçóes de ótimo verdadeiro.

Os sistemas multivariados fundamentados em planejamento fatorial de experimentos permitem avaliar este tipo de interação, o que favorece estudos de otimização, a partir de um reduzido número de experimentos.

\section{REFERÊNCIAS}

APHA, Standart Methods for Examination of Water and Wastewater, 19a ed., Amer. Publ. Ass: Washington, 1995.

BARROS NETO, B., SCARMINIO, I. S., BRUNS, R. E. Como fazer experimentos: pesquisa e desenvolvimento na ciência e na industria. Editora da Unicamp, 2001.

CHEMKEYS, http://www.chemkeys.com/bra/ $\mathrm{md} /$ peeo_6/mdoeq_1/mdpf_3/mdpf_3.htm, acessado em abril de 2005 .

DUEÑAS, M. et al. Exopolysaccharide production by Pediococcus damnosus 2.6 in a semidefined medium under different growth conditions. International Journal of Food Microbiology, v. 87, n. 1-2, p. 113-120, 2003.

GALETAKIS, M.; RAKA, S. Utilization of limestone dust for artificial stone production: an experimental approach. Minerals Engineering, v. 17, n. 2 , p. $355-357,2004$.

HARDY, A. et al. A statistical approach to the identification of determinant factors in the preparation of phase pure $(\mathrm{Bi}, \mathrm{La})_{4} \mathrm{Ti}_{3} \mathrm{O}_{12}$ from an aqueous citrate gel. Journal of the European Ceramic Society, v. 24, n. 9, p. 2575-2581, 2004.

LEGRINI, O.; OLIVEROS, E.; BRAUN, A.M. Photochemical processes for water treatment. Chem. Rev., v. 93, p. 671-698, 1993.

KOCHANA, et al. Synthesis of standards of the most important markers of Leuckart p-methoxymethamphetamine (PMMA): Examination

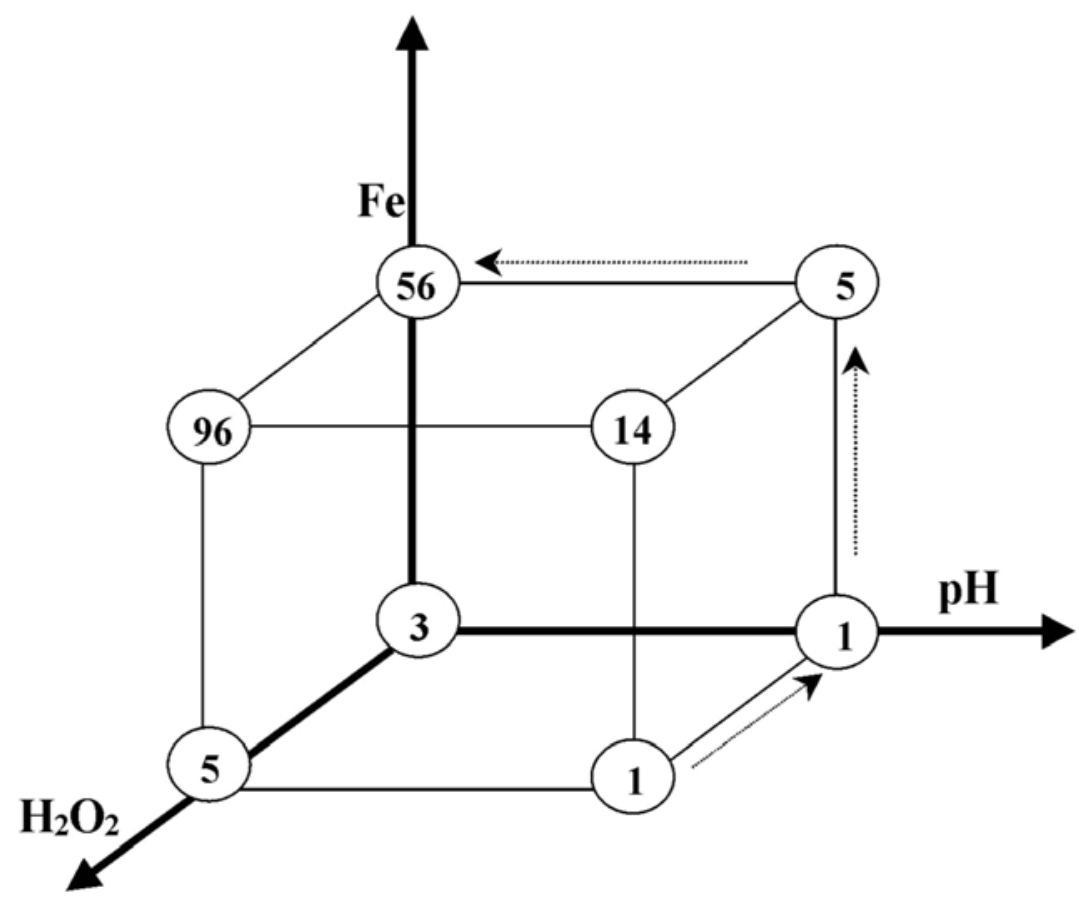

Figura 3 - Representação geométrica do planejamento fatorial $2^{3}$ para otimização das condições de degradação do corante Laranja Reativo 16 por processo envolvendo ferro metálico (Resposta: \% de degradação do corante avaliada por espectroscopia UV-Vis)

of the influence of experimental conditions and a drug diluent on SPE/TLC profiling. Forensic Science International, v. 134, n. 2-3, p. 207-213, 2003.

MONTES, P.; BREMNER, T.W.; LISTER, D.H. Influence of calcium nitrite inhibitor and crack width on corrosion of steel in high performance concrete subjected to a simulated marine environment. Cement and Concrete Composites, v. 26, n. 3, p. 243-253, 2004.

SHARP, W.G.; HARGROVE, D.S. Emotional expression and modality: an analysis of affective arousal and linguistic output in a computer vs. paper paradigm. Computers in Human Behavior, v. 20, n. 4, p. 461-475, 2004.

WANG, Z-P. et al. Landfill leachate treatment by a coagulation-photooxidation process. Journal of Hazardous Materials, v. 2887, p. 1-7, 2002.

YI-ZENG, L.; KAI-TAI, F.; QING-SONG, $\mathrm{X}$. Uniform design and its applications in chemistry and chemical engineering. Chemometrics and Intelligent Laboratory Systems, v. 58, n. 1, p. 43-57, 2001.
Endereço para correspondência:

Patricio Peralta-Zamora

Departamento de Química Universidade Federal do Paraná Caixa Postal 1908 I

8153 I-990 Curitiba - PR - Brasil

Tel.: (4I) 336I-32-97

Fax: (4I) 336I-3I 86

E-mail: zamora@quimica.ufpr.br 